\title{
Histoire de la santé publique et communautaire en Afrique. Le rôle des médecins de la mission suisse en Afrique du Sud ${ }^{1 *}$
}

Hines Mabika

\section{Summary}

It was not Dutch settlers nor British colonizers who introduced public and community health practice in north-eastern South Africa but medical doctors of the Swiss mission in southern Africa. While the history of medical knowledge transfer into $19^{\text {th }}-20^{\text {th }}$ century Africa emphasises colonial powers, this paper shows how countries without colonies contributed to expand western medical cultures, including public health. The Swiss took advantage of the local authorities' negligence, and implemented their own model of medicalization of African societies, understood as the way of improving health standards. They moved from a tolerated hospital-centred medicine to the practice of community health, which was uncommon at the time. Elim hospital's physicians moved back boundaries of segregationist policies, and sometime gave the impression of being involved in the political struggle against Apartheid. Thus, Swiss public health activities could later be seen as sorts of seeds that were planted and would partly reappear in 1994 with the ANC-projected national health policy.

Keywords: South Africa, Switzerland, Public Health, Medical Missions, Medical Apartheid.

\footnotetext{
* L'auteur remercie les Profs V. Barras et. P. Gisel pour le soutien financier de leurs institutions lors de la rédaction de cet article dans le cadre de la coopération interfacultaire de l'Université de Lausanne, le Prof. H. Steinke (Berne) pour ses amicales suggestions et les deux éditeurs anonymes pour la pertinence de leurs commentaires. La recherche fut conduite dans le cadre du projet SNF sur l'histoire des systèmes de santé en Afrique, dirigée par les Profs P. Harries et B. Obrist de l’Université de Bâle, également remerciés.
}

Hines Mabika, Institut für Medizingeschichte der Universität Bern, Bühlstrasse 26, CH-3012 Bern (hines.mabika@img.unibe.ch) 


\section{Résumé}

Ce ne furent ni les occupants d'origine hollandaise ni les colonisateurs britanniques qui introduisirent les pratiques de santé publique et communautaire dans le nord-est de l'Afrique du Sud mais des médecins de la mission suisse. Alors que le transfert des savoirs médicaux en Afrique s'énonce habituellement au crédit d'anciennes puissances coloniales, cet article démontre que des pays sans colonies à l'instar de la Suisse, participèrent à l'expansion de la culture médicale occidentale. Ils allèrent à la rencontre des communautés villageoises sous le régime de l'apartheid et leur dispensèrent des notions d'éducation sanitaire et de prévention contre des pathologies. Ils expérimentèrent ainsi leur propre modèle de médicalisation des sociétés locales et reculèrent les barrières des politiques ségrégationnistes, donnant l'impression d'un positionnement politique face au régime d'apartheid. Une sorte de graine de santé publique communautaire semée par ces médecins parut resurgir en 1994 dans le cadre du projet de politique nationale de santé du Congrès National Africain (ANC).

\section{Introduction}

Si la médecine missionnaire est le «parent pauvre» des travaux d'histoire de la médecine coloniale, la santé publique missionnaire l'est encore davantage. ${ }^{1}$ Elle n'a pas encore mobilisé d'études significatives à l'heure où l'histoire de la médecine en Afrique devient un champ très actif. Des travaux portent davantage sur le passé des services publics de santé mis en place par les puissances coloniales européennes (Grande Bretagne et France). ${ }^{2}$ Ils traitent de l'histoire des maladies. ${ }^{3}$ Les efforts des missionnaires sont relégués au second rang; ceux des pays sans colonies ignorés. Printz Othon tenta une mise en lumière des activités médicales des Suisses et des Alsaciens de la société des missions évangéliques de Paris dans la région de Lambaréné en Afrique centrale avant la Première Guerre mondiale. ${ }^{4}$ Au nord-est du Transvaal en Afrique du Sud, la mission du Canton de Vaud servit de matrice à la médecine communautaire. ${ }^{5}$ A près la fondation de l'hôpital d'Elim en 1899 par Georges

1 Faure 2012; Harrison/Jones/Sweet 2009; Digby 2006; Good 2004.

2 Crozier 2007; Echenberg 2002; Domergue-Cloarec 1986.

3 Curtin 1998; Ranger/Slack 1992; Hartwig/Patterson 1978.

4 Printz 2004.

5 Deux thèses sont en cours sur les activités médicales des missions de Bâle (Pascal Schmid, Université de Bâle) et des Capucins de Lucerne (Marcel Dreier, Université de Bâle). La recherche postdoctorale de l'auteur à l'Université de Bâle porta sur la mission vaudoise (cf. ses publications de 2009, 2011, 2012 et 2013). 
Liengme (1859-1936) du Jura bernois, ses compatriotes et successeurs vaudois ou bâlois y introduisirent des pratiques de santé publique et communautaire, soixante années plus tard. Cet article montre comment les Suisses en sont venus à y pratiquer la santé publique au sens d'«une science et un art de la prévention de la maladie, du prolongement de la vie et de la promotion de la santé à travers l'organisation des efforts de la société». ${ }^{6}$ L'organisation des efforts sanitaires qu'ils mirent sur pied dans les communautés villageoises de ces contrées fit de la santé publique un rempart contre la dégradation de la condition sanitaire et sociale. Cette étude explore le rôle des médecins de la mission dans un contexte politique binaire polarisé par la question de la ségrégation médicale. Elle évoque non seulement la capacité du médecin missionnaire du $\mathrm{XX}^{\mathrm{e}}$ siècle à défier parfois l'ordre politique, mais surtout ses initiatives relevant des pratiques de santé publique et communautaire à l'heure où le paradigme des soins de santé primaire issu de la conférence d'Alma Ata de 1978 n'était pas encore d'actualité. ${ }^{7}$

\section{Pratiquer la biomédecine?}

Lorsque les missionnaires suisses fondèrent leur première station missionnaire du nord-est du Transvaal, le 9 juillet $1875,{ }^{8}$ leur intention n'était pas de pratiquer la biomédecine. Le but n'était pas de soigner les corps des populations africaines, mais de conquérir leurs âmes et, ainsi, agrandir la bergerie du Dieu chrétien. L'établissement des missions médicales suisses s'inscrivait dans un contexte international constitué par le débat autour de la nécessité de joindre la sanctification de l'âme à la purification du corps. ${ }^{9}$ Les principales sociétés des missions protestantes de Suisse (Bâle, 1815; Vaud, 1875) développèrent respectivement la Basler Ärztliche Mission ${ }^{10}$ et la Commission médicale de la mission vaudoise dans l'Afrique du sud. ${ }^{11}$ Cette dernière avait son siège à Lausanne et visait à résoudre la question du manque d'intérêt des populations dites païennes pour l'évangile. Les pionniers des missions évangéliques avaient reçu une formation médicale, complémentaire de leurs

6 OMS 1991.

7 Porter 1994.

8 Bulletin de la mission vaudoise 18,3 (1875). Ce Bulletin devint ensuite le Bulletin de la mission suisse. Il était adressé par la Commission des missions de l'Eglise évangélique libre du Canton de Vaud aux souscripteurs pour la mission vaudoise: T.1, n ${ }^{\circ} 1$ (1872)-T.4 (1883), $\mathrm{n}^{\circ} 51(1955)$.

9 Gelfand 1984.

10 Fischer 1991.

11 Grandjean 1917. 
études théologiques. ${ }^{12}$ Ernest Creux et Paul Berthoud tirèrent profit de ce complément de formation en offrant quelques soins médicaux aux populations de leurs stations missionnaires du Transvaal dès la fin des années $1870 .{ }^{13}$ La mission vaudoise (1875), devenue mission romande (1883) avec l'adhésion des déléguées des cantons de Neuchâtel et de Genève au Conseil des missions des Eglises libres du canton de Vaud, prit la dénomination de «mission suisse romande dans le sud de l'Afrique» (MSAS) en 1918, et depuis 1963, de «Missions et Echanges».

Le véritable tournant vint du financement des études médicales de Georges Liengme par les Eglises Libres du Canton de Vaud au début des années 1880. Cette pension assura la mission de disposer d'un premier médecin qualifié. ${ }^{14}$ Liengme devint en effet le premier médecin-missionnaire de Suisse romande. Il venait du Jura Bernois où il naquit à Courtelary en 1858, et étudia la médecine à Berne et Genève avant d'être consacré le 12 juillet 1891 à l'Oratoire de Genève. Il servit ensuite dans les actuelles républiques du Mozambique (1891-1895) et d'Afrique du Sud (1895-1906) avant de fonder, en 1908, une des premières institutions rurales de psychiatrie, près de Vaumarcus à la frontière des cantons de Neuchâtel et de Vaud où il mourut en 1936. Quant à la mission de Bâle, elle envoya Rudolph Fischer en Côte de l'Or (Ghana) en 1885 dans le cadre de sa nouvelle mission médicale. Ces entreprises en médecine apportèrent une aide vigoureuse à leurs activités évangéliques. Elles recadrèrent et renforcèrent les méthodes et moyens d'évangélisation des peuples non chrétiens. Cet objectif dessinait déjà une forme d'instrumentalisation de la science. La médecine apparut comme un outil au service de l'évangélisation, et le médecin missionnaire un allié de premier plan de l'expansion culturelle et économique qu'on qualifia de mission civilisatrice. ${ }^{15}$

En ces temps, le Transvaal constituait depuis la convention de Sand River de 1852 une république boer, la Zuid Afrikanische Republiek des descendants d'Hollandais. ${ }^{16}$ Ces derniers s'y étaient installés une vingtaine d'années plus tôt, en provenance du Cap - sud du pays - devenu en 1806 une colonie britannique. Les Suisses qui évangélisèrent le nord du Transvaal venaient du Lesotho où ils avaient fait leurs premiers pas de missionnaires au sein de la

12 Harries 2007a.

13 Bulletin de la mission suisse 1919; Archives du DMR: E. Creux et P. Berthoud reçurent respectivement un an et trois ans de formation médicale. L'offre de soins de santé était prioritairement destinée à leurs compatriotes missionnaires puis aux populations locales des régions respectives d'Elim, Valdézia, Laurenço Marques ou de Pretoria.

14 Archives du Château de Penthes: Lettres de G. Liengme.

15 Conklin 1997, 39.

16 Ross 2008, 52 et 74-90. 
société des missions évangéliques de Paris. ${ }^{17}$ Au Transvaal, les destinataires de l'évangile étaient davantage préoccupés par la recherche des solutions à leurs misères temporelles que par la promesse d'une jouissance éternelle dans un paradis échappant à leur cosmogonie, comme l'ont montré les Comaroff dans leur analyse de la rencontre entre les Tswana et les missionnaires. ${ }^{18}$ Une réponse pratique au pragmatisme local fut donnée par l'inauguration en 1899 de l'hôpital missionnaire du petit village d'Elim à 400 kilomètres au nord de Pretoria, dans l'une des régions les plus pauvres du pays. Les débuts de cet hôpital cö̈ncidèrent avec la dernière guerre anglo-boer (1899-1902) qui transforma le Transvaal en colonie britannique en 1902. La création de l'Union Sud-Africaine en 1910 (Cap, Transvaal, Natal et Etat d'Orange) consacra l'union ethnique (Whites only) qui adopta le Native Land Act en 1913. Cette loi attribuait 93 puis $87 \%$ du territoire à la minorité d'origine occidentale et abandonnait 7 puis $13 \%$ du territoire aux $80 \%$ de la population constituée par les Africains. Ce contexte historique prépara le terrain à la conceptualisation de la doctrine d'apartheid et son application géographique, économique et politique dès $1948 .{ }^{19} \mathrm{Ce}$ fut dans ce cadre politique et juridique tendu qu'émergea et s'affirma la médecine missionnaire suisse et ses pratiques ultérieures de santé publique et communautaire. Mais comment se positionna cette médecine missionnaire des temps coloniaux?

L'historiographie africaniste coloniale et postcoloniale se distança dès les années 1970 de l'opposition entre une histoire triomphaliste de la médecine et de la santé et une histoire plus revendicative, dont les partisans du marxisme furent de prolifiques auteurs dès les indépendances africaines. ${ }^{20}$ Certes, la césure est plus doctrinaire qu'empirique, et la réalité factuelle permet souvent de tempérer les discours vantant les apports de la colonisation médicale. ${ }^{21}$ La tendance est désormais de constater la diversité des fortunes en fonction de l'organisation des systèmes et des politiques de santé accoudées à l'organisation des territoires et aux réalités conjoncturelles. La carence des travaux d'histoire de la médecine missionnaire en contexte colonial n'est guère proportionnelle à son importance dans les activités des pays qui avaient étendu leur domination culturelle, économique et/ou politique en Afrique aux $\mathrm{XIX}^{\mathrm{e}}-\mathrm{XX}{ }^{\mathrm{e}}$ siècles. ${ }^{22}$ Le développement de sa pratique se fit à l'aune de

17 Mabika 2013,2-4.

18 Comaroff/Comaroff 2004,181-197.

19 Ross 2004; Worden 1994; Bonner/Delius/Posel 1993; Posel 1991.

20 Vaughan 2007; Mbokolo 1984.

21 Audoynaud 2010; Lapeysonnie 1988.

22 Crozier 2007; Bado 2007; Domergue-Cloarec 1986.

Gesnerus 72 (2015) 
l'essor des sociétés de missions, elles-mêmes résultant des stratégies hiérarchisées. ${ }^{23}$ La médecine missionnaire représenta bien un des canaux d'introduction des sciences médicales occidentales en Afrique. Son approche diachronique et analytique invite à repenser le concept même de médecine coloniale, entendue davantage comme la médecine officielle et publique de l'administration coloniale. ${ }^{24}$ Qu'elle fût aux mains de l'autorité publique ou des acteurs confessionnels, la médecine en Afrique du Sud fut un instrument d'expansion culturelle et d'influence spirituelle des populations autochtones. Sous l'apartheid, elle devint même un puissant instrument de contrôle social et de domination raciale. ${ }^{25}$ La quête de sens ne procède pas seulement de ce que cette médecine fut synchrone de la pratique médicale coloniale, mais aussi de la porosité des frontières décelable entre ces médecines. En effet, si l'une était réputée publique et laïque, et l'autre, privée et confessionnelle, des relations financières tutélaires entre l'administration locale coloniale et postcoloniale et la médecine missionnaire suisse semblaient abattre certaines frontières. ${ }^{26} \mathrm{Ce}$ positionnement rend la médecine missionnaire comptable de la diffusion de la culture médicale occidentale. Bien qu'elle ne fût pas une puissance coloniale, la Suisse prit une part active dans les activités de cette expansion culturelle et économique européennes en Afrique, participant de l'idéologie colonialiste analysée par l'historien fribourgeois Patrick Minder. ${ }^{27}$ En plus de construire et de véhiculer l'idée que l'on se faisait de l'Afrique, les activités des ressortissants et organisations suisses et leur consécutive influence étaient importantes. La forte influence économique et financière des entreprises suisses en Afrique du sud que vient de rappeler l'ouvrage de Sandra Bott s'inscrivait dans ce discret activisme suisse à la limite de l'invisible et cependant puissant. ${ }^{28} \mathrm{Au}$ nord-est du Transvaal, l'engagement pour l'introduction des pratiques de santé publique et communautaire fut tel qu'on en vint à évoquer l'hypothèse de la santé publique comme le champ politique de la médecine missionnaire suisse au $\mathrm{XX}^{\mathrm{e}}$ siècle.

23 A l'instar de la mission vaudoise opérant au nord-est du Transvaal, d'autres missions suisses furent actives dans le domaine de la médecine en Afrique: la mission de Bâle était présente au Ghana et au Cameroun; les Capucins de Lucerne en Tanzanie.

24 Domael 1997.

25 Horwitz 2009; Fassin 2004; Marks 1994; Marks/Trapido 1993; Price 1984.

26 Voir deux articles à paraître de l'auteur: «Histoire du financement de la santé en Afrique: les hôpitaux missionnaires suisses du nord-est du Transvaal» et «Le docteur Georges Liengme, un médecin aux frontières?».

27 Minder 2011. Cette monographie ne traite pratiquement pas de la médecine missionnaire suisse mais soutient la thèse d'une participation des Suisses «à un esprit colonial communément partagé en Europe».

28 Bott 2013. 
Si la pratique depuis la fin du $\mathrm{XIX}^{\mathrm{e}}$ siècle de la biomédecine dans les régions considérées visait à attirer les populations locales à la chrétienté, ni le Révérend Ernest Creux et ni Paul Berthoud et encore moins le docteur Georges Liengme n'envisagèrent ces développements. Creux et Berthoud tenaient des infirmeries informelles dans leurs logements de missionnaires. Liengme exerça d'abord à Shiluvane avant d'aller renforcer les activités médicales du Révérant Creux à Elim. ${ }^{29}$ Même après avoir remplacé Creux en 1896, Liengme dut attendre le développement de ces activités pour envisager la construction de l'hôpital d'Elim. ${ }^{30}$ Cela devint possible avec la création en Suisse d'un fonds d'investissement - la Société Immobilière - qui permit ensuite de réunir le capital nécessaire au projet hospitalier. Le projet prit forme dès avril 1898 avec les débuts de construction de l'hôpital inauguré en novembre 1899.

Photo 1: Bâtiment principal de l'hôpital missionnaire suisse à Elim, 1975. ${ }^{31}$

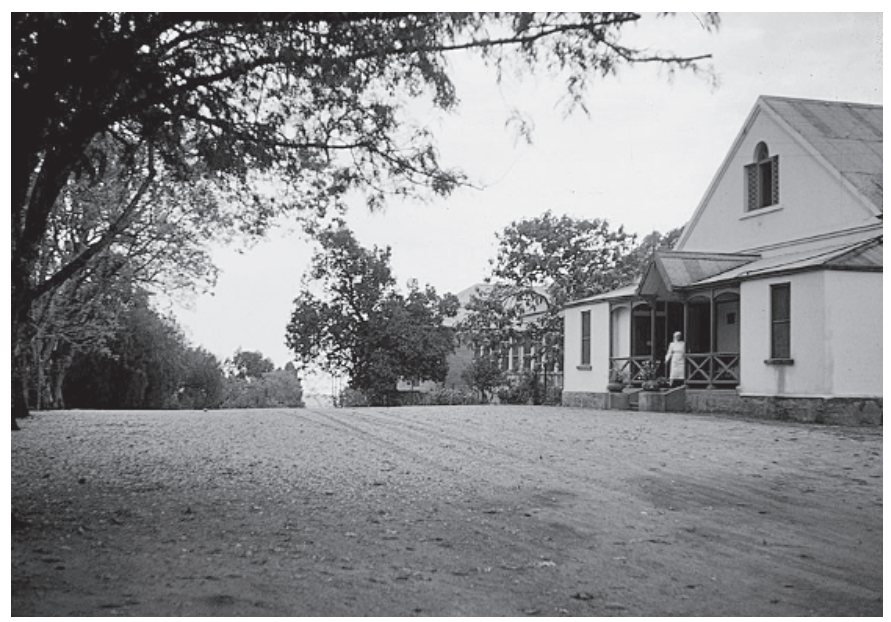

Lorsque des incompréhensions avec la hiérarchie missionnaire poussèrent Liengme à démissionner de son poste en 1906, il fut tout de suite remplacé par son compatriote, le docteur James Borle, arrivé l'année précédente à Elim. Ce dernier succomba plus tard des suites de l'épidémie de grippe espagnole en 1918 alors qu'il venait de s'installer à son propre compte à Johannesburg. Il avait dès la veille de la Première Guerre mondiale cédé sa place au docteur Max de Legniris qui acquit, pour l'hôpital, le premier équipement de radiologie et de Rayons X en 1912. Cette petite révolution technologique pour cet hôpital rural du début du $\mathrm{XX}^{\mathrm{e}}$ siècle africain amorça la modernisation de son plateau technique qui s'étoffait au fil des ans. On pro-

29 Staehelin 2008.

30 Liengme 1906.

31 Les photographies contenues dans cette contribution proviennent des archives privées du Dr Erika Sutter. Elles ont récemment été déposées à la Basler Afrika Bibliothek et référencées sous la côte BPA60. 
céda désormais à la radiographie des parties du corps comme les membres ou le thorax, et diagnostiqua des fractures osseuses et des pathologies comme la tuberculose étudiée dans le pays par Randall Packard. ${ }^{32}$

La pratique médicale missionnaire suisse n'était pas isolée. Elle composait le système de santé du Transvaal et plus largement celui de l'Afrique du Sud comme entité politique. Un système non uniforme dans lequel coexistaient plusieurs microsystèmes fondés sur la race, le statut privé ou public, le lieu de vie, etc. La mission suisse était ainsi soumise aux administrations sanitaires provinciale et centrale, et respectait la ségrégation raciale en tant que «vieille idée qui n'avait de nouveau que le terme apartheid». ${ }^{33}$ L'hôpital adopta le Public Health Bill, ${ }^{34}$ une loi édictée par Pretoria au lendemain de l'épidémie de grippe espagnole d'octobre $1918 .{ }^{35}$ Cette norme attribuait au gouvernement central la compétence pour des questions relevant des épidémies et des maladies contagieuses (notifiables diseases). En clair, elle permettait aux hôpitaux de bénéficier des subventions du gouvernement au prorata des cas de contagions traités et d'épidémies endurées tout en finançant des politiques en ces matières.

En dehors du capital de construction de l'hôpital de 132000 francs suisse constitué en Europe par la Société Immobilière, la mission médicale ne put jamais s'autofinancer. Le défaut d'une recette suffisante se doubla d'un déficit des ressources humaines. Cela eût constitué un véritable frein à l'action médicale missionnaire si l'aide financière annuelle de 400 livres accordée par la Province dès 1902 n'avait pas augmenté au fil des ans pour atteindre, en 1948, un subside de 13138 livres britanniques contre 10761 livres de recettes d'honoraires et de dispensaire et 68 livres de dons et de divers. ${ }^{36}$ Au soutien de l'administration du Transvaal s'ajoutait l'aide du gouvernement central de Pretoria et des dons individuels et collectifs de Suisse et d'Afrique du Sud. Les contributions publiques se justifiaient par l'intention missionnaire d'attirer les populations à la chrétienté. Cette orientation enchantait l'autorité locale qui, d'une part, en escompta un certain dessaisissement de l'Etat de ses devoirs régaliens, et d'autre part, une civilisation des populations locales, c'est-à-dire leur soumission aux nouveaux modes de pensée, de fonctionnement et de vie. ${ }^{37}$ Le second aspect témoignant de l'intégration de l'hôpital missionnaire suisse dans le dispositif du système national de santé est l'adhésion d'Elim aux pratiques d'organisation des hôpitaux sud-africains

32 Packard 1989.

33 Mandela 1994, 111.

34 Giyani Archives: Public Health Bills, 1919.

35 Phillips 1990.

36 Elim Hospital Archives: Annual Report, Finances, 1948, 3.

37 Comaroff/Comaroff 2004. 
de l'époque, fondées sur des entrées séparées pour les patients africains et pour les patients d'ascendance occidentale, bien que le respect de ces dispositions ne signifiât pas leur cautionnement. Contrairement aux principes en vigueur, Max de Legneris projeta la formation d'un personnel auxiliaire de santé local. Il saisit l'occasion de l'émotion suscitée par les ravages de la grippe espagnole pour initier la formation d'un personnel africain au sein de l'hôpital. Faute de volonté de la part de l'administration locale, l'initiative traîna en longueur et n'aboutit qu'en $1932 .{ }^{38}$ La témérité du médecin de la mission fit de son hôpital l'un des rares lieux où les autochtones pouvaient bénéficier d'une formation sanitaire comme à l'hôpital McCord du Natal. Cet investissement en ressources humaines s'ajoutait à l'équipement de l'institution et à la construction de nouveaux bâtiments, comme celui de la maternité en 1938 ou encore le pavillon des malades indiens commencé en 1947 et inauguré en 1949. Si le pavillon des patients indiens avait été financé par la communauté indienne, l'essentiel des financements de l'hôpital provenait des subsides publics. ${ }^{39}$

\section{Initier des pratiques de santé publique}

A la veille de la victoire électorale du National Party qui décréta le régime de l'apartheid en 1948, le rapport de la Commission Gluckman (1944-1946) sur le système de santé dans l'Union sud-africaine révéla des inégalités sanitaires inacceptables. Les résolutions introduisaient le concept de centre de santé comme structure sanitaire de base à l'échelle du pays. Mais l'une des premières décisions prises par le nouveau régime fut le rejet des résolutions dudit rapport. Or, à cette époque déjà,la MSAS fondait justement sa pratique médicale sur un système missionnaire de soins dans lequel l'hôpital d'Elim jouait un rôle central. Des infirmeries ou clinics servaient de portes d'entrée dans ce système de soins dans un rayon de vingt à trente kilomètres. Dans les années 1950-1960, cette infrastructure missionnaire était relativement importante. Outre l'hôpital d'Elim, elle comprenait les infirmeries de Valdezia, Shiluvane, Khurulene et Tlangelane. Ces formations sanitaires étaient périodiquement visitées par le médecin d'Elim. Les cas cliniques critiques étaient dirigés vers Elim.

Les réticences populaires à la médicalisation des sociétés étaient peu notables, à moins de se référer aux rapports, au départ, peu enthousiastes de

38 Nos Nouvelles médicales 1 (1934).

39 Elim Hospital Archives: Buildings, 1900-1948.

Gesnerus 72 (2015) 
certaines populations avec la mission médicale en Afrique du Sud comme ailleurs en Afrique. ${ }^{40} \mathrm{Il}$ s'agissait dans ces cas des populations attachées aux pratiques locales sur le plan médical et religieux. Or, la médecine missionnaire œuvrait à l'arrachement des populations de ce qu'on qualifiait de paganisme. L'expression des réserves par certains membres de la MSAS quant au succès des activités médicales en marge du sacerdoce missionnaire l'évangile ou la mission spirituelle - constitua une difficulté initiale au Transvaal. Des débats minaient la mission médicale de l'intérieur et portaient à la fois sur l'interprétation du rapport entre le spirituel et le temporel, ainsi que sur la réalité des carences multiformes. Ils privèrent la mission médicale d'un véritable soutien financier interne. Si à Lausanne et sur le terrain africain, quelques membres de l'Eglise ne voyaient que perte d'énergie et de moyens dans une activité séculière, fût-elle médicale, le marasme sanitaire et social local, et les hésitations des populations africaines face à l'évangile conduisirent les missionnaires à forger des stratégies de pénétration de ce milieu.

L'offre conjointe des soins du corps et des soins de l'âme entrait dans cette démarche stratégique. Elle s'imposa sur le terrain et permit de glisser de la pratique curative vers une pratique davantage préventive. Dès les années 1960 en effet, des études épidémiologiques révélaient une détérioration de la condition sanitaire des populations locales depuis au moins une vingtaine d'années. Conséquemment, la prévention des maladies et l'éducation sanitaire furent progressivement initiées par les médecins de la mission suisse dès le milieu de la décennie suivante. Ces initiatives participaient aussi de la nécessité pratique de contourner des carences financières, matérielles et humaines chroniques de la pratique médicale hospitalière suisse dans la région. Elles permettaient d'impliquer les populations dans la gestion de leur santé et questionnaient de fait les concepts de santé et santé publique. La notion de prévention renvoyait ici à une préoccupation épistémologique relative au concept de santé publique, comme l'entendait Charles Edward Winslow en 1920, en insistant effectivement sur la prévention. Pour le bactériologiste américain, en effet, le but de la santé publique était

la prévention des maladies, la prolongation de la vie et la promotion de la santé et de l'efficacité physiques par des efforts coordonnées de la communauté pour l'assainissement de l'environnement, le contrôle des infections dans la population, l'éducation de l'individu aux principes de l'hygiène personnelle, l'organisation des services médicaux et infirmiers pour le diagnostic précoce et le traitement préventif des pathologies [...]. ${ }^{41}$

Ainsi, il convient d'inclure non seulement la prévention mais aussi le traitement préventif des pathologies, même si la nocivité de certains traitements

40 Langwick 2011; Digby 2006, Ch. 3/8; Hunt 1999.

41 Winslow 1920. 
préventifs pour les personnes saines est désormais mieux établie. ${ }^{42} \mathrm{Si}$ les acceptions ultérieures de la discipline, notamment celle, somme toute récente, proposée par l'OMS, inclut l'organisation des soins curatifs, l'expérience des missionnaires médicaux suisses au nord du Transvaal montre que l'introduction des pratiques de santé publique fut un processus. Celui-ci incluait des études, des traitements préventifs, l'éducation et la promotion sanitaires. L'histoire de l'introduction de ces pratiques en Afrique du Sud ne résulta cependant pas d'un déroulement linéaire, mais participa d'un contexte international favorable et d'une réalité locale dynamique marquée par des tensions générationnelles au sein de la mission médicale suisse et d'une conjoncture politique particulière.

\section{Le contexte international de l'introduction de la santé publique}

L'introduction des pratiques de santé publique dans les villages du nord-est sud-africain fut quasiment synchrone de l'émergence de la médecine communautaire dans plusieurs régions du monde: sud des Etats-Unis, Europe de l'Est (notamment URSS), mais aussi Chine et divers pays africains comme la Tanzanie, le Nigéria ou le Sénégal. ${ }^{43}$ Le contexte international de bipolarisation née de la guerre froide n'en était pas la seule explication. Le fait s'inscrivait dans un long mouvement de globalisation des politiques et pratiques de santé. George Rosen nota que l'idée d'organiser des campagnes systématiques en vue de maîtriser certaines catégories de maladies et de déséquilibres commença d'essaimer dès le début du $\mathrm{XX}^{\mathrm{e}}$ siècle aux Etats-Unis à travers l'éducation sanitaire des communautés. ${ }^{44} \mathrm{La}$ situation fut différente en Europe où le National Health Service Act de l'ancien colonisateur britannique adopté en 1946 n'entra en vigueur qu'en 1948. C'est plutôt l'URSS qui disposait d'un des systèmes d'éducation sanitaire communautaire les plus aboutis au début de la deuxième moitié du $\mathrm{XX}^{\mathrm{e}}$ siècle, avec son Institut central de recherche en éducation sanitaire de Moscou qui forma plus de 2000 médecins de santé publique entre 1950 et $1952 .{ }^{45}$ Ce contexte eut sans doute un effet sur les positions de l'autorité sud-africaine, qui voyait en la pratique de la médecine communautaire une velléité communiste poten-

42 Une étude écossaise présentée à la société européenne de cardiologie en 2009 révéla les risques liés à la prise préventive de l'aspirine contre des attaques cardiaques. La nocivité pour la santé résiderait surtout dans des risques d'hémorragie. http://sante.lefigaro.fr/actualite/ 2009/09/01/9745-nouvelle-mise-garde-contre-laspirine.

43 King 1966; Morley 1973.

44 Rosen 1958, 392.

45 Rosen 1958, 403.

Gesnerus 72 (2015) 
tiellement subversive. En faisant avorter les résolutions de la Commission Gluckman de 1944-1946, le régime de l'apartheid confirma l'absence de véritable volonté d'unifier le système de santé national malgré l'Union politique et économique datant de $1910 .{ }^{46} \mathrm{Or}$, si le concept de «soins de santé primaire» a une connotation assez différente selon que nous parlons des pays en développement ou des pays industrialisés, ${ }^{47}$ il se réfère néanmoins à l'approche épidémiologique des problèmes de santé, à leur prévention par l'implication des communautés et à l'éducation et la promotion sanitaires telle que la développa la Chine de Mao Zedong avec son système de formation des paysans aux rudiments de médecine dans le cadre des médecins aux pieds nus dans les années $1960 .{ }^{48}$ L'approche des médecins de la mission suisse, surtout à partir du milieu des années 1970, s'inscrivait donc dans ce contexte international d'émergence de la médecine communautaire et reliait de ce fait des initiatives d'une région d'Afrique à des stratégies sanitaires pensées à l'échelle globale.

\section{Renouvellement générationnel et la naissance des tensions}

Ce fut l'arrivée d'une nouvelle génération des médecins de la MSAS, jeunes et libérés d'une sorte de positivisme doctrinaire du tournant des années 1950, qui impulsa une nouvelle dynamique médicale à Elim dans un contexte d'apartheid facteur d'ethnicité. ${ }^{49}$ Parmi ces nouvelles recrues figurait Gabrielle Guye de Neuchâtel. Elle naquit au Mozambique en 1916 de parents missionnaires neuchâtelois. Formée à la pratique des soins infirmiers à l'Institution La Source à Lausanne (1937-1941) où elle obtint son diplôme d'infirmière, elle travailla à Genève, Lausanne et Neuchâtel avant de s'initier à la didactique des soins infirmiers en Grande Bretagne. Ce parcours fut essentiel pour améliorer la formation des infirmières autochtones d'Elim commencée dans l'entre-deux-guerres. Gabrielle Guye devint, en effet, responsable de l'école d'infirmières d'Elim entre 1947 et 1978, et ne rentra en Suisse qu'en 1978, profitant d'une longue retraite avant de décéder à Neuchâtel le $1^{\text {er }}$ janvier 2012.

Erika Sutter, née à Bâle en 1917, était l'une de ces jeunes recrues. Docteur en biologie botanique, elle arriva à Elim en 1952 âgée de 35 ans. Elle œuvra au service du laboratoire de radiologie et Rayons X de l'hôpital avant de

46 Mill/Michel/Kreeb 1999, 41.

47 Groenevegen/van der Zee 1990, 89-90.

48 Wikki 2004.

49 Harries 2007b; Horrel 1973. 
s'inscrire en faculté de médecine à Johannesburg et obtenir le $M B C h B$, équivalent d'un doctorat en médecine de l'Université de Wits en 1962. Elle servit comme médecin à Elim dès 1965, revint se spécialiser en chirurgie oculaire à Bâle, en médecine tropicale à Londres, puis repartit servir à Elim. Elle prit la retraite en 1984 et rentra à Bâle où elle vit toujours. ${ }^{50}$

Photo 2: Dr Erika Sutter à la remise des prix, $\mathrm{CG}$ du Village Bugeni, 1984.

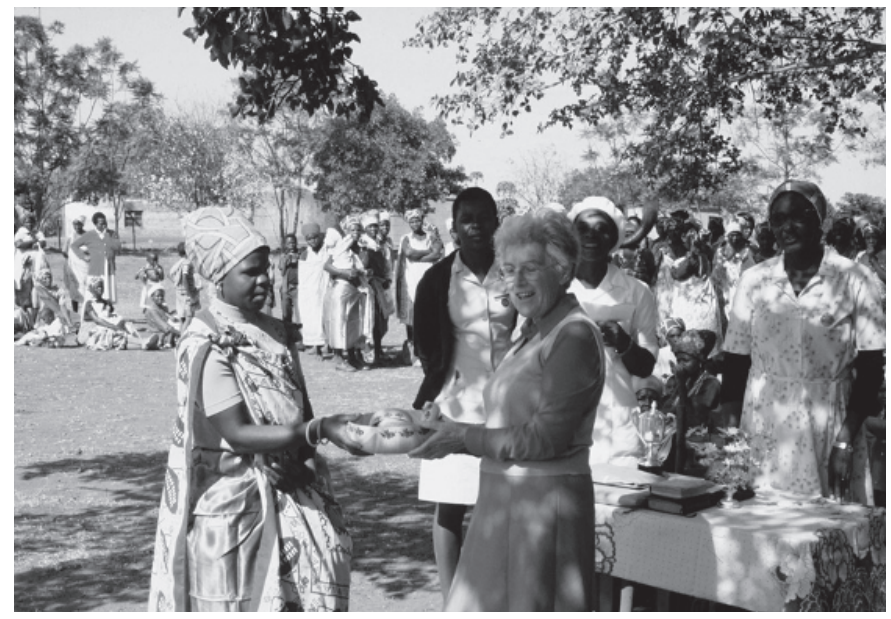

Peter Kok et R. Verhage, deux jeunes médecins originaires de Hollande, vinrent également servir à Elim pendant cette période de renouvellement générationnelle de la mission médicale suisse. Ils furent parmi les chevilles ouvrières de l'introduction des pratiques de santé publique et communautaire à l'hôpital et ses environs. Ils s'étaient initiés à deux aspects majeurs des soins de santé primaire à l'Université de Groningen au Pays-Bas: la médecine de famille et la médecine communautaire. Aussi furent-ils à l'origine des premières enquêtes épidémiologiques relatives à la malnutrition infantile autour d'Elim.

Les initiatives des médecins de la MSAS suscitèrent des tensions au sein de l'hôpital mais aussi entre l'hôpital et l'administration locale. Elles furent parfois perçues comme un positionnement et un engagement politiques potentiellement subversifs. C'est ainsi que l'administration déroula une stratégie de contrôle des activités de la mission médicale. Il s'agissait d'exercer un pouvoir indirect sur les populations. Le soutien financier à l'hôpital fut augmenté, tout en exigeant de ce dernier un compte rendu de ses activités. Dans le même temps, le gouvernement s'affaira à conceptualiser et faire mettre en place sa «Comprehensive Health Service», une politique de santé censée intégrer les aspects de médecine curative, préventive et de promotion de la santé, également dans une visée de contrôle social et politique.

50 Stiehle 2011.

Gesnerus 72 (2015) 
Ce personnel de santé de l'hôpital suisse s'inscrivit hors des cadres fixés par l'autorité locale. Il créa son propre cadre opératoire et fit recours à des pratiques de santé alors inhabituelles dans la région: enquêtes sanitaires, implication des communautés ou encore éducation sanitaire. Pour utiles qu'étaient ces pratiques, elles servirent aussi l'image de la mission et donnèrent de ces médecins une réputation embellie de l'Occident dans une Afrique postcoloniale revendicative de libertés et d'une autonomie longtemps confisquées. En ce sens, l'idée de simple bonté, justificative desdites entreprises, est à déconstruire. Tout en inscrivant ces initiatives dans leurs devoirs de médecins ruraux, elles renforcèrent leur expérience en santé publique et communautaire.

\section{Etudes épidémiologiques et éducation sanitaire}

Cette nouvelle génération de professionnels de la santé à Elim ne se cantonna pas à la pratique hospitalière. Elle engagea des recherches sur le terrain et impliqua les communautés villageoises en leur expliquant le bien-fondé de cette participation. Cette posture était contraire à celle prônée par la direction de l'hôpital, qui encourageait une certaine distance avec les communautés locales, garantissant de la sorte de bons rapports de l'institution sanitaire avec l'administration locale et plus largement avec le régime de l'apartheid. Les nouveaux médecins tinrent au contraire à visiter les communautés dans leurs villages et à conduire des enquêtes épidémiologiques. Selina Maphorogo et Erika Sutter, dans leur ouvrage, Community is my University, rapportent le propos du docteur Kok:

C'est en étant confronté à des enfants présentant divers stades de malnutrition que nous décidâmes de conduire une recherche. Nous souhaitions comprendre d'où venaient tous ces enfants dont $60 \%$ souffraient de malnutrition, et quelles en étaient les causes. Nous pensions que les enfants reçus en consultation à l'hôpital ne devraient représenter qu'une infime partie d'une population malade plus nombreuse qui n'aurait pas accès aux soins. ${ }^{51}$

L'autorisation et l'aide demandées à la direction de l'hôpital pour conduire une enquête épidémiologique au sein de la population furent refusées. Ceci s'explique par le fait que la législation initiée par le régime dès 1949 interdisait aux Européens de fréquenter des lieux d'habitation des Africains. ${ }^{52}$ Les rapports patient-médecin devaient se limiter au colloque médical dans le cadre de la clinique hospitalière. De plus, la relation de la direction de l'hôpital avec l'administration locale était différente de celle que pouvaient avoir 
des jeunes médecins arrivés d'Europe au lendemain de la Seconde Guerre mondiale avec un pouvoir jugé raciste. Leurs projets d'enquêtes épidémiologiques apparurent comme un positionnement politique justifiant la recherche de solutions aux problèmes des populations. Ils ne s'en tinrent donc pas au refus du docteur Pierre Jaques, directeur de l'hôpital, et entreprirent ces études pour leur propre compte: «Nous nous servions de nos véhicules privés et conduisions ces travaux pendant notre temps de repos (samedis après-midi)».$^{53}$ Près de 300 enfants furent examinés dès la première expérience. Les inquiétudes exprimées tantôt par les médecins se révélèrent fondées: la malnutrition était un problème grave. Les enfants en sous-poids représentaient $22 \%$ des enquêtés; parmi ceux-ci, $3 \%$ présentaient un état de malnutrition sévère et $1 \%$ des enfants étaient atteints de kwashiorkor (malnutrition sévère par défaut d'alimentation protéinée).

Outre les études épidémiologiques, leur engagement hors des murs de l'hôpital consista en l'éducation sanitaire des populations au travers de la diffusion des notions élémentaires de santé publique et communautaire dès 1972. Les études entreprises avaient permis de reconstituer la chaine causale des malnutritions. La carence en viande dans l'alimentation des enfants reçus à l'hôpital provenait notamment du fait que les populations ne se nourrissaient plus de poulets domestiques - une épizootie en étant la cause. Le recours à un vétérinaire de la ville voisine de Louis Trichardt aida à enrayer le malheur. De plus, ces populations purent bénéficier de rudiments d'enseignement leur permettant de procéder eux-mêmes à l'immunisation des poules. Le docteur Sutter rappelle que la formation des populations aux techniques d'immunisation des poules éveilla la curiosité de ces dernières et les amena à comprendre la malnutrition de leurs enfants et les possibilités de la prévention des maladies. Des notions d'hygiène du corps et du milieu leur étaient désormais dispensées pour prévenir les problèmes de santé. Ce paradigme de santé publique se compléta avec la création du concept des groupes de soins communautaires par les médecins de la mission: les Care Groups (CGs) dès le milieu des années 1970.

En effet, outre la malnutrition, le trachome - infection oculaire due au chlamydia trachomatis - constitua un important problème de santé publique dans la région. Cette affection entrait pour un tiers dans les causes de cécité à l'âge adulte. Elle partageait cette responsabilité avec le glaucome et la cataracte. Conscients de la possibilité de prévenir le trachome, les médecins de la mission s'attaquèrent à ce qu'ils percevaient comme la «racine» du problème: le défaut d'hygiène - un autre problème de santé publique en soi.

53 Sutter, entretien du 6 février 2009.

Gesnerus 72 (2015) 
Ce nouvel engagement déboucha sur le développement d'un projet de lutte contre ladite affection. Cette fois, la direction de l'hôpital apporta son soutien au projet en lui affectant une infirmière, une assistante de soins et un travailleur social. Cette évolution de l'attitude de la hiérarchique de l'hôpital s'expliquait sans doute par le constat de détermination des acteurs et l'enthousiasme suscité par les premiers résultats. L'équipe dénommée The Trachoma Team reçut par la suite des formations en santé publique et permit une mutation du concept de santé publique qui, désormais, développait véritablement son côté communautaire en intégrant la participation des membres des communautés villageoises dans la prévention de ce problème de santé et des préoccupations connexes. L'initiatrice du projet, l'oculiste bâloise Erika Sutter, avait précédemment participé à une campagne du Council for the Blind en faveur des écoliers du nord Transvaal. Fort des limites constatées dans cette campagne, à laquelle les parents, notamment les mères des écoliers n'étaient pas associées, le projet Trachoma s'engagea non seulement à travailler avec les femmes mais surtout à leur concéder un rôle central. On aboutit dans plusieurs villages à la création des groupes de soins ou CGs. Dans le fonds, cette approche communautaire avait quelques précédents en Afrique: les expériences de Maurice King au Kenya et David Morley au Nigeria témoignaient de la circulation des pratiques de santé en Afrique. ${ }^{54} \mathrm{Il}$ s'agissait davantage d'un transfert vertical du nord vers le sud, de l'Europe vers l'Afrique, que de transfert horizontal d'un pays africain à un autre. Dans leurs ouvrages parus dans les années 1960-1970, King et Morley décrivent leurs pratiques expérimentales, désormais reconnues comme liées à la santé publique et communautaire. ${ }^{55}$ Nombre de ces pratiques héritées de la médecine de campagne en Occident et transférées en Afrique étaient réappropriées plus ou moins différemment selon les territoires, les peuples mais aussi les rapports historiques entre les acteurs en présence.

Les Care Groups du nord-est du Transvaal consistaient en groupes de femmes œuvrant à l'amélioration des conditions de santé et de vie dans leurs communautés villageoises. A l'image des paysans chinois qui reçurent de la part des médecins des rudiments de savoir médical, les femmes dites Care Groups Fellows furent formées aux bonnes pratiques de santé et devinrent des agents bénévoles de la médicalisation des sociétés locales, au sens de

54 La période coloniale vit l'avènement des mesures d'hygiène dans la plupart des régions sous domination occidentale. Mais les pratiques de préservation et promotion de la santé introduites en zones rurales dans les années 1960-1970 furent d'un genre nouveau et impliquaient davantage les communautés.

55 King 1966; Morley 1973. 
l'introduction de la tradition médicale occidentale dans ces sociétés. Pour autant, l'entreprise de glissement du pouvoir sanitaire de l'institution hospitalière vers les communautés ne fut aisée. Le premier essai de travail communautaire organisé en 1975 dans le village de Raphahlelo fut un échec. La mésaventure était due au fait que le Trachoma Team ne travaillait pas directement avec les communautés, mais avec leurs représentants: le chef du village, l'infirmière ainsi que d'autres élites rurales. ${ }^{56}$

Une première étude de 1976 dans les villages de Chavanu, Nkuzane et Mbokota révéla au docteur Sutter et son équipe la nécessité et la portée du travail directe avec les communautés. De même, une connaissance précise de la prévalence moyenne de la pathologie $-50 \%$ chez des enfants de 2 à 5 ans - était nécessaire. Le défaut d'hygiène comme cause de propagation de l'infection oculaire déjà énoncée apparut clairement. Les enfants des familles les plus pauvres étaient plus affectés que ceux des élites villageoises, telles qu'instituteurs, infirmières, notables. Sutter et ses collègues rendirent compte des résultats de leurs recherches dans le South African Medical Journal. ${ }^{57}$ Ils démontrèrent en substance que les enfants scolarisés étaient les meilleurs propagateurs de l'affection au sein des familles; l'éducation sanitaire des mères aurait un impact sur leurs enfants; elle fragiliserait la chaine de propagation de la maladie dans les foyers. Ainsi, l'observation des règles d'hygiène du corps et du milieu, l'utilisation d'une lavette de bain par membre de la famille et non plus une lavette pour tous limiterait la propagation de la maladie; enfin, une intervention sur la condition économique des populations permettrait même de l'enrayer.

Ces conclusions provoquèrent un élan d'activisme contre le cercle vicieux de la pauvreté et de la cécité due au trachome. L'action se focaliser désormais sur la transformation de la condition socio-économique des populations villageoises pour une meilleure santé. Les $C G s$ symbolisaient cette aspiration et organisaient des March towards a better future.

L'hôpital consentit à fournir des moyens humains plus importants, et tendit même à s'approprier l'initiative. Un service de santé publique fut initié au sein de l'hôpital. Le docteur Peter Kok en devint le référent jusqu'à sa nomination au Secrétariat Général du Ministère de la Santé créé en 1976 dans le Bantoustan du Gazankulu. Ce parcours participait de la nouvelle donne née de la nationalisation de l'hôpital missionnaire suisse en 1976 et de l'opportunité offerte aux employés de la mission médicale suisse de

56 Entretien avec Selina Maphorogo à Waterfall, Elim Hospital, Afrique du Sud, juillet 2008 et septembre 2010.

57 Sutter/Ballard 1978. 




Photo 3: Des membres des CG sur le terrain - marche vers un meilleur avenir, années 1980.

continuer de servir dans n'importe quel hôpital d'Afrique du sud ou de démissionner. Après avoir offert ses services au ministère de la santé de Gazankulu, Kok partit pour le Kenya en 1979, où les approches de médecine communautaire et de santé publique prenaient également leur essor.

Les $C G s$ de la région d'Elim consistaient en groupes de 10 à 100 femmes formées aux notions d'éducation sanitaire lors des réunions convoquées par l'équipe de Sutter, désormais renforcée par des infirmières, aides-soignantes et travailleurs sociaux. Cette équipe était chargée de rencontrer les communautés villageoises et de les motiver à adopter des réflexes de santé publique et communautaire. Ces motivateurs jouaient un rôle majeur dans la transmission des savoirs et pratiques des soins élémentaires de santé aux membres des dits CGs. En amont des motivateurs demeuraient les responsables du projet, les médecins. Les connaissances et pratiques de soins diffusées venaient en majorité d'eux. Elles comprenaient les postures à adopter devant la maladie ou tout problème de santé. Ces connaissances, pratiques, et postures de santé étaient traduites dans des chants, des Care Groups Songs.

Sutter prit sa retraite en 1984, le docteur Carl Ijsselmuiden du département de santé publique de l'hôpital d'Elim reprit la direction du projet des $C G s$ avant de laisser à son tour la responsabilité à Selina Maphorogo. Ijsselmuiden était d'origine hollandaise et suivit les traces de nombreux compatriotes engagés dans la mission médicale en Afrique du Sud. Il avait rejoint les effectifs de l'hôpital d'Elim en 1983 et passa un diplôme de santé publique à l'école de médecine de Medunsa au Transvaal en 1984. L'aide-soignante Maphorogo s'était distinguée au poste de «Motivatrice» par son charisme et son aptitude à rallier les communautés villageoises à l'esprit et aux pratiques des CGs. 
Photo 4: Equipe des infirmières - motivatrices, 1979.

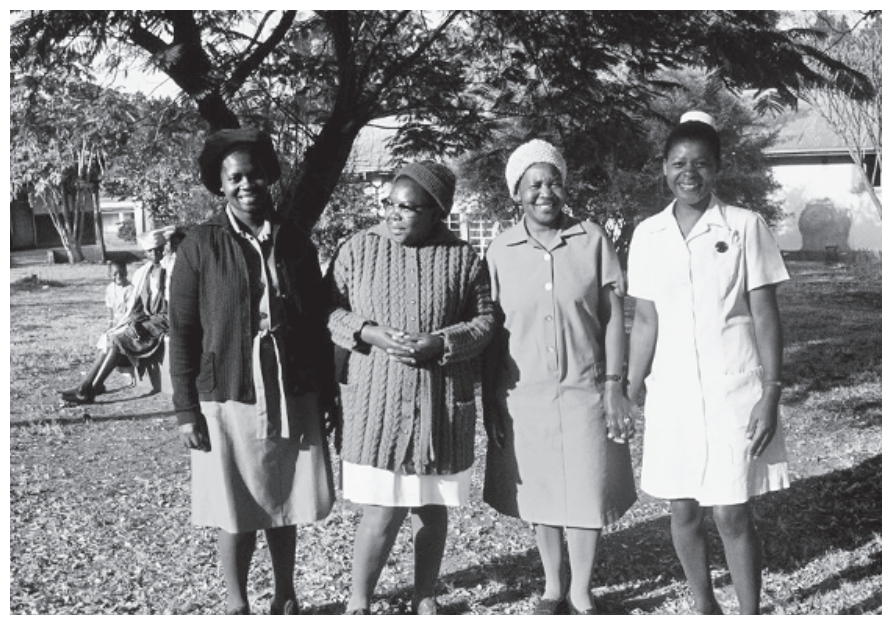

Les savoirs et pratiques véhiculés reposaient sur la participation des membres de ces communautés à la prévention des maladies, l'éducation sanitaire et l'amélioration de l'hygiène corporelle et environnementale. La santé publique était ainsi entendue à la fois comme une démarche de quête du bienêtre physique et psychosocial et des pratiques d'amélioration des conditions d'existence communautaire. Les populations apprirent à s'organiser individuellement et collectivement, et développèrent des projets économiques, à l'instar des jardins de culture maraîchère derrière des habitations, ou encore la réalisation des latrines et la fabrication de briques de terre utilisables dans la construction d'habitats.

Ces initiatives ainsi que l'application locale des principes et normes élaborés ou suggérés par les médecins d'Elim ou leurs collaborateurs n'allaient pas sans poser quelques problèmes pratiques à l'extérieur comme au sein de l'hôpital. Si, à l'extérieur, la témérité des acteurs médicaux avait poussé la hiérarchie à admettre leurs interventions hors des murs de l'hôpital, l'apartheid médical au sein de l'hôpital demeurait une question importante. Il supposait, entre autres, que les patients d'origine européenne fussent aux soins des infirmières blanches et non des auxiliaires africaines. Or, non seulement les effectifs des infirmières missionnaires suisses ne permettaient pas leur affectation exclusive au service des patients sud-africains blancs, mais leur vocation missionnaire était de «soigner les pauvres Africains et non pas des sales boers». ${ }^{58}$ La formation d'un personnel local à Elim se justifiait par ce déficit. La professionnalisation du domaine infirmier et son remodelage à l'échelle nationale par des contingences politiques et ethniques des classifications distinguèrent non seulement le personnel africain de celui d'origine

58 DMR Archives, Lausanne, 8. Correspondances. Nombreuses lettres et des rapports en provenance d'Elim mentionnent ce sujet dès les années 1920. 
occidentale, mais encore l'infirmière d'origine anglaise de l'Afrikaner. ${ }^{59}$ Ces classifications traduisaient des velléités de domination corollaire d'une relation de pouvoir complexe, actrice de violence. Cette violence occultée est aussi paradigmatique de ce que l'introduction de la santé publique en Afrique n'est habituellement étudiée que sur l'angle unilatéral des apports des seules anciennes grandes puissances coloniales. Or, les missions médicales constituèrent d'autres canaux d'émergence des pratiques de santé publique. L'étude de l'internationalisation de ces pratiques offre ainsi une autre perspective de genèse de la santé publique et communautaire en Afrique. ${ }^{60}$ Les traces de ces réalisations constituent des objets de mémoire de plus en plus reconnus en Afrique du sud. Dans ce sens, l'hôpital d'Elim a acquis en 1996 le statut de monument national. Deux ans auparavant, le projet de politique sanitaire national de l'ANC publié en 1994 faisait du centre de santé l'unité de base du système de santé. Or, une vingtaine d'années auparavant, cette disposition existait de facto dans les villages du nord-est du pays dans le cadre du système rural de soins de la mission suisse. Ce rappel historique n'est pas sans rapport avec l'actuelle politique mémorielle faisant de l'hôpital d'Elim un objet de célébrations. La célébration en 1999 du centenaire de sa fondation draina dans le petit village d'Elim, un public nombreux venu de toute l'Afrique du Sud et de l'étranger. ${ }^{61}$ Ce qui sembla compter fut «non plus les déterminants mais leurs effets; non plus les actions mémorisées ni même commémorées, mais la trace de ces actions et le jeu de ces commémorations; pas les événements pour eux-mêmes, mais leur construction dans le temps, l'effacement et la résurgence de leurs significations [...]», ${ }^{62}$ expression au Transvaal d'une sorte d'engagement des médecins de la mission suisse. L'analyse des discours des acteurs de cette mission révèle le sens de cet engagement, au-delà d'une simple attitude morale du missionnaire chrétien. Ce positionnement sembla transcender la seule posture déontologique médicale et éthique: «Nous pensions que c'était à nous de le faire (d'introduire les pratiques de santé publique dans ces régions pauvres et relativement délaissées par le gouvernement), et nous l'avons fait, car nous n'étions pas d'accord avec le régime». ${ }^{63} \mathrm{Ce}$ propos de la docteure bâloise semble présenter l'initiative d'introduction des pratiques de santé publique au nord du Transvaal comme une réalisation dont la mission médicale suisse fut la matrice.

59 Sud-africaine d'origine hollandaise, généralement née en Afrique, et parlant une langue non européenne: l'Afrikaans.

60 Litsios 2004.

61 Elim Hospital Archives: Elim Centenary, 1999.

62 Nora 1993.

63 Sutter, entretien, Bâle, 6 février 2009. 


\section{Conclusion}

La médecine missionnaire suisse contribua à l'introduction des pratiques de santé publique et communautaire en Afrique du Sud. Au service de l'entreprise d'évangélisation des populations autochtones, le soin du corps devait attirer les populations locales à la foi chrétienne, et préfigurer la sanctification de l'âme. Cette sorte d'instrumentalisation de la science à des fins d'influence culturelle connut des développements imprévus au moment de l'institutionnalisation de l'apartheid médical dans le pays. L'arrivée à Elim d'une nouvelle génération des médecins - jeunes et intéressés par une approche communautaire de la santé - changea la donne. En effet, la menace de disparition des populations locales sous les effets de l'apartheid médical dès les années 1950 coïncida avec l'initiative des médecins de la mission suisse d'agir au-delà de l'hôpital. Cette initiative suscita des tensions au sein de l'hôpital - avec la hiérarchie -, mais aussi à l'extérieur - avec l'autorité publique opposée aux rapports interraciaux et, partant, à l'implication des communautés villageoises dans l'organisation de la lutte contre leurs problèmes de santé. La témérité des médecins opéra malgré tout et tendit à inverser les courbes statistiques de la morbidité individuelle et collective. Conséquemment, bien avant la Conférence d'Alma Ata de 1978 qui donna de l'allant aux soins de santé primaire, des études épidémiologiques précédèrent des groupements de soins communautaires dénommés Care Groups. Des notions d'hygiène du corps et du milieu ainsi que des rudiments d'économie sociale furent dispensés. Des affections comme le trachome, responsable de plus d'un tiers des cas de cécité à l'âge adulte, et diverses misères corollaires purent être freinées, puis quasiment éradiquées. Ces démarches de santé publique participaient d'une volonté de conjurer les misères occasionnées ou aggravées par l'apartheid. La convocation des approches de soins de santé primaire dans le projet politique de l'ANC en 1994 remit le rôle des missions en général, et particulièrement celui de la mission médicale suisse, au centre des débats sur les questions de santé publique en Afrique du sud. L'implication des communautés sous la supervision des agents de l'hôpital initiée par ladite mission médicale redevient la norme dans le contexte général de déficit des personnels de santé. ${ }^{64} \mathrm{~S}$ 'il est vrai que le paradigme du droit de chacun à la santé offre un espace d'intervention aux médecins de la mission suisse ${ }^{65}$ participant à la mutation d'une médecine hospitalière et curative vers une approche communautaire préventive des maladies, il est permis aussi de convenir avec Didier Fassin que «pour ceux qui y participent

64 Schneider/Lehmann 2009.

Gesnerus 72 (2015) 
comme malades, thérapeutes ou décideurs», ${ }^{66}$ la santé publique est aussi et avant tout un objet politique au quotidien.

\section{Archives}

Archives du Château de Penthes, Genève, Suisse

Archives du Département Missionnaire, Lausanne, Suisse

Archives and Musée de l'Hôpital d'Elim, Afrique du Sud

Basler Afrika Bibliothek, Bâle, Suisse, notamment le fonds photographique BPA 60.

\section{Bibliographie}

Audoynaud, André, Eloge de la médecine coloniale: regard sur la santé en Afrique (Paris 2010)

Bado, Jean-Paul, Les conquêtes de la médecine moderne en Afrique (Paris 2007)

Bonner, Philip/Peter Delius/Deborah Posel, Apartheid's Genesis, 1935-1962 (Johannesburg 1993)

Bott, Sandra, La Suisse et l'A frique du Sud, 1945-1990: marché de l'or, finance et commerce durant l'apartheid (Zürich 2013).

Bulletin de la mission Vaudoise (Périodique de la mission vaudoise dans l'Afrique du Sud,1872-1955, dénommé aussi Bulletin de la mission suisse romande, Bulletin de la mission suisse dans l'Afrique du Sud).

Comaroff, Jean/John Comaroff, Of Revelation and Revolution. Christianity, Colonialism and Consciousness in South Africa (Chicago ${ }^{4} 2004$ )

Conklin,Alice L., A Mission to Civilise, the Republican Idea of Empire in France, 1895 1930 (Stanford 1997)

Crozier, Anna, Practising Colonial Medicine: the Colonial Medical Service in British East Africa (London 2007)

Curtin, Philip, Disease and Empire. The Health of European Troops in the Conquest of Africa (Cambridge 1998)

Digby, Anne, Diversity and Division in Medicine: Health Care in South Africa from the 1800s (Oxford 2006)

Domergue-Cloarec, Danielle, Politique coloniale française et réalités coloniales. La santé en Côte d'Ivoire, 1905-1958 (Toulouse 1986)

De Certeau, Michel, L'écriture de l'histoire (Paris 1975)

Echenberg, Myron, Black Death, White Medicine. Bubonic Plague and the Politics of Public Health in Colonial Senegal, 1914-1945 (Portsmouth N.H. 2002)

Ewald, François, L'Etat providence (Paris 1986)

Fassin, Didier (éd.), Afflictions. Afrique du Sud, de l'apartheid au sida (Paris 2004)

Fassin, Didier, «La santé, un enjeu politique», in: Politique Africaine 28 (1987), 2-8

Faure, Olivier (éd.), Missions religieuses, missions médicales et «mission civilisatrice» (XIX $X^{e}$ et $X X^{e}$ siècles) (Paris 2012)

Fischer, Friedrich Hermann, Der Missionsarzt Rudolf Fisch und die Anfänge medizinischer Arbeit der Basler Mission an der Goldküste (Ghana) (Herzogenrath 1991)

65 London/Schneider 2012.

66 Fassin 1987. 
Gelfand, Michaël, Christian Doctor and Nurse. The History of Medical Missions in South Africa (Johannesburg 1984)

Grandjean, Arthur, La mission romande: Ses racines dans le sol suisse. Son épanouissement dans la race tsonga (Lausanne 1917)

Groenevegen P.P./J. van der Zee, «Organisation and Research Program of the Netherlands Institute of Primary Health Care», in: Bergerhoff, Petra/Dieter Lehmann/Peter Novak (eds.), Primary Health Care: Public Involvement, Family Medicine, Epidemiology and Health Economics (Berlin 1990)

Good, Charles, The Steamer Parish: the Rise and Fall of Missionary Medicine on an African Frontier (Chicago 2004)

Harries, Patrick et al., SNIS Final Report: Working Paper Research Project <History of Health Systems in Africa> (2011) (http://www.snis.ch/system/files/working paper_a_history_of_health_system.pdf, 26.2.2015)

Harries, Patrick, Butterfliers \& Barbarians. The Swiss Mission System of Knowledge in South-Sastern Africa (Oxford 2007a)

Harries, Patrick, «Exclusion, Classification and Internal Colonialism:The Emergence of Ethnicity among the Tsonga-Speakers of South Africa», in:Leroy, Vail, The Creation of Tribalism in Southern Africa, (Berkeley etc. 2007b) 82-117

Harrison, Mark/Margareth Jones/Helen Sweet (eds.), From Western Medicine to Global Medicine: The Hospital beyond the West (Hyderabad 2009)

Hartwig, Gerald W./David K. Patterson (eds.), Disease in African History: An Introductory Survey and Case Studies (Durham, 1978)

Horrel, Muriel, The African Homelands of South Africa (Johannesburg 1973)

Horwitz, Simonne, Health and Health Care under Apartheid (Johannesburg 2009)

Hugon, Anne, Un protestantisme africain au $19^{e}$ siècle: l'implantation du méthodisme en Côte de l'Or, 1835-1874 (Paris 2007)

Jeannerat, Caroline/Eric Morier-Jenoud/Didier Péclard, Embroiled: Swiss Churches, South Africa, and Apartheid (Zürich 2011)

King, Maurice, Medical Care in Developing Countries. A Primer on Medicine of Poverty and a Symposium from Makerere (London 1966)

Langwick, Stacy L., Bodies, Politics, and African Healing. The Matter of Maladies in Tanzania (Bloomington 2011)

Liengme, Georges, Un Hôpital Sud-Africain (Neuchâtel 1906)

Litsios, Socrates, «The Christian Medical Commission and the Development of World Health Organization's Primary Health Care Approach», in: Am J Public Health 94 (2004) 1884-1893

London, Leslie/Helen Schneider, «Globalisation and Health Inequalities: Can a Human Rights Paradigm Create Space for Civil Society Action?», in: Social Science and Medicine, 74/1 (2012) 6-13.

Luke, Francis Richard, Health in Our Time. A Plea (Johannesburg 1946)

Mabika, Hines, «Religion et Politique dans le Bulletin de la mission suisse dans le sud de l'Afrique, 1872-1955», in: Revue suisse d'histoire religieuse et culturelle, 106 (2012) 85-106

Mabika, Hines, «La mission médicale suisse au sud de l'Afrique, 1896-1976», in: Mosimann-Barbier, Marie-Claude/Michel Prum (éd.), Colonialisme et Colonialisme: Le Lesotho à l'heure du bicentenaire d'Eugène Casalis (Paris 2013) 167-184 
Mandela, Nelson: Long Walk to Freedom. The Autobiography of Nelson Mandela (New York 1994)

Maphorogo, Selina/Erika Sutter/Jennifer Jenkins (eds.), Community is My University. A Voice from the Grass Roots on Rural Health and Development (Amsterdam 2003)

Marks, Shula, Divided Sisteerhood: Race, Class, and Gender in the South African Nursing Profession (Johannesburg 1994)

Marks, Shula/Stanley Trapido, The Politics of Race, Class, and Nationalism in Twentieth-Century South Africa (London 1993)

Mill, Thomas R./Alexander Michel/Martin M. Th. Kreeb (eds.), Public Health in Südafrika. Beiträge zu einem Gesundheitssystem im Wandel (Lage 1999)

Minder, Patrick, La Suisse coloniale: les représentations de l'Afrique et des Africains en Suisse au temps des colonies, 1880-1939 (Bern 2011)

Morley, David, Peadiatric Priorities in the Developing World (Boston 1973)

Nora, Pierre, Les lieux de mémoire (Paris 1993). Nouvelles de nos missions médicales, 1934-1963 (Supplément médical du Bulletin de la mission Suisse paraissant dès 1934).

Packard, Randall M., White Plague, Black Labor: Tuberculosis and the Political Economy of Health and Disease in South Africa (Berkley 1989)

Porter, Dorothy, The History of Public Health and the Modern State (Amsterdam 1994)

Posel, Deborah, The Making of Apartheid (Oxford 1997)

Price, Max, «Health Care as an Instrument of Apartheid Policy in South Africa», in: Health Policy and Planning, 1, 2 (1986) 158-170

Printz, Othon, Avant Schweitzer, les génies tutélaires de Lambaréné: Robert Nassau, Valentine Lantz, Maurice Robert (Colmar 2004)

Ranger, Terence/Paul Slack (eds.): Epidemics and Ideas. Essays on the Historical Perception of Pestilence (Cambridge 1992)

Ross, Robert, A Concise History of South Africa (Cambridge 2008)

Schneider, H./U. Lehmann, «Lay Workers and HIV Programmes: Implications for Health Systems», in: AIDS Care, 22, 1 (2010) 60-67.

Scott, J.G, «Trachoma in the South African Bantu», S Afr Med J, 24 (1986) 355

Seedat, Aziza, Crippling a Nation. Health in Apartheid South Africa (London 1984)

Staehelin, Sarah, A Swiss Mission Hospital in the Transvaal (Basel 2008)

Stiehle, Gertrude, Erika Sutter: Mit anderen Augen gesehen. Erinnerungen einer Schweizer Augenärztin (Basel 2011)

Sutter, Erika/R.C. Ballard, «A Community Approach to Trachoma Control in Northern Transvaal», in: S. Afr. Med. J. 58 (1978) 622-625.

Van Domael, Monique, «La médecine coloniale ou la tradition exogène de la médecine moderne dans le Tiers Monde», in: Studies in Health Services Organisation and Policy, 1 (1997) 1-38.

Winslow, Charles Edward, «The untilled field of Public Health», in: Science (1920) 2333.

Wikki, Valentine, Health for the Masses: China's Barefoot Doctors, (online publication 2005, http://www.npr.org/templates/story/story.php?storyId=4990242, 2.3.2015)

Worden, Nigel, The Making of Apartheid. Conquest, Segregation and Apartheid (Oxford 1994) 